\title{
Nefrolitiasi da iperparatiroidismo primitivo: uso del Cinacalcet in pazienti non trattabili con terapia chirurgica
}

\author{
M. Terribile ${ }^{1}$, A. Giliberti ${ }^{1}$, M. Capuano ${ }^{1}$, R. Rossano, S. Sisca ${ }^{2}$, M. Lombardi ${ }^{2}$ \\ ${ }^{1}$ U.O.C. di Nefrologia e Dialisi e Centro Calcolosi Renale, Ospedale dei Pellegrini, Napoli \\ ${ }^{2}$ Nefrologia e Dialisi, Ospedale S.M. Annunziata, Azienda Sanitaria di Firenze
}

\section{Introduzione}

L’iperparatiroidismo (IPTP) primitivo è la più comune causa di ipercalcemia. Nell' $85 \%$ dei casi è presente un singolo adenoma. Nel 10\% dei casi vi è una iperplasia ghiandolare e infine nel 5\% dei casi si riscontra un carcinoma o più adenomi.

L'IPTP comporta una aumentata secrezione dell'ormone paratiroideo (iPTH), cui consegue un'alterazione del metabolismo calcio-fosforo e quindi un aumentato rischio di nefrolitiasi e di demineralizzazione ossea. L'IPTP è normalmente trattato mediante paratiroidectomia. Tuttavia l'approccio chirurgico non è sempre possibile a causa di gravi patologie concomitanti, di pregresso insuccesso chirurgico, di dislocazione anomala delle ghiandole stesse, di rifiuto da parte del paziente.

Talvolta l'intervento chirurgico non è neanche pienamente appropriato a causa di una patologia asintomatica o di lieve entità, o di IPTP con assenza di progressione. In tali casi vi è pertanto controversia circa la necessità di un intervento chirurgico.

Fino ad alcuni anni orsono non vi erano alternative alla paratiroidectomia per ridurre i livelli di calcemia e di PTH. Da alcuni anni è disponibile un'altra opzione terapeutica, rappresentata dai calcio-mimetici. I calciomimetici e il Cinacalcet (C) in particolare, sono molecole che attivano il calcium-sensing receptor e inibiscono la secrezione di PTH (1). Sono chiamati calciomimetici perché mimano gli effetti del calcio extracellulare, attivando il recettore del calcio e inibendo le funzioni delle cellule paratiroidee (2). Il C è comunemente ed efficacemente utilizzato nella terapia dell'IPTP secondario alla insufficienza renale cronica $(3,4)$. Da circa 3-4 anni vi sono riscontri positivi circa il suo utilizzo anche nell'IPTP come efficace alternativa terapeutica al trattamento chirurgico.

Riportiamo i casi clinici di due pazienti con IPTP non trattabile con terapia chirurgica sottoposti a terapia con C.

\section{Casi clinici}

\section{Caso 1}

Paziente S.M. di anni 64. Giunge alla nostra osservazione nel gennaio 2003. Egli fa riferimento al nostro Centro per la Calcolosi per una storia clinica di nefrolitiasi protratta nel tempo. Il paziente aveva precedentemente eliminato 9 calcoli urinari: 4 espulsi spontaneamente e 5 in seguito a trattamenti di litotripsia extracorporea.

L'ecografia renale evidenziava 2 calcoli a dx e 3 calcoli a sn (diametro tra i 6 e i $9 \mathrm{~mm}$ ).

È stato pertanto da noi effettuato lo studio metabolico che evidenziava la presenza di IPTP: calcemia 11.8 $\mathrm{mg} \%$, iPTH $130 \mathrm{pg} / \mathrm{mL}$ e calciuria $450 \mathrm{mg} /$ die. Il paziente presentava normofunzione renale con creatininemia $0.9 \mathrm{mg} \%$ e clearance della creatinina (Clcr) 70 $\mathrm{mL} / \mathrm{min}$. È stato inoltre effettuato un esame MOC femore che evidenziava osteoporosi con $\mathrm{T}$ score $>-2.5$ DS.

È stata quindi da noi prescritta terapia idropinica + ci- 


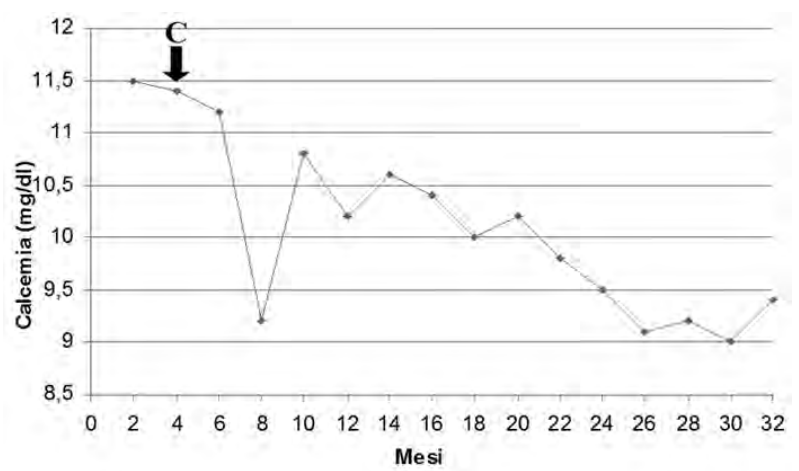

Fig. 1 - Effetti del Cinacalcet (C) sui livelli sierici di Ca durante il periodo di studio.

TABELLA I - CRITERI CLINICI E LABORATORISTICI PER PARATIROIDECTOMIA NELL'IPTP, NIH GUIDELINES 2002 (5)

$\rightarrow$ Calcolosi renale recidivante

$\rightarrow$ Calcemia $>1 \mathrm{mg} / \mathrm{dL}$ rispetto ai limiti della norma

$\rightarrow$ Calciuria $24 \mathrm{~h}>400 \mathrm{mg}$

$\rightarrow$ Clearance della creatinina $<30 \%$ della norma

$\rightarrow$ MOC T score $<-2.5$ DS

$\rightarrow$ Età $<50$ anni

*La presenza di almeno uno di questi criteri può rappresentare una indicazione clinica per la paratiroidectomia

trati + difosfonati.

Nell'aprile 2003 il paziente è stato sottoposto a ecografia delle paratiroidi: "nella norma". Successivamente ha praticato scintigrafia paratiroidea con sestamibi: "non evidenza di tessuto paratiroideo iperfunzionante".

Nel giugno 2003 ha effettuato TAC cervicotoracica con mdc: "nella norma" e RMN cervico toracica: "nella norma".

Il paziente è stato quindi sottoposto a visita specialistica di endocrino chirurgia. Essendo presenti 4 criteri clinici e laboratoristici per paratiroidectomia indicati dalle Linee Guida (Tab I), è stato proposto un intervento di chirurgia esplorativa che il paziente ha rifiutato.

Pertanto è continuato il trattamento con terapia idropinica + citrati e disfosfonati.

Nel corso dei successivi 3 anni il paziente ha prodotto altri 4 calcoli.

Nel maggio 2006 il paziente ha consultato un'altra

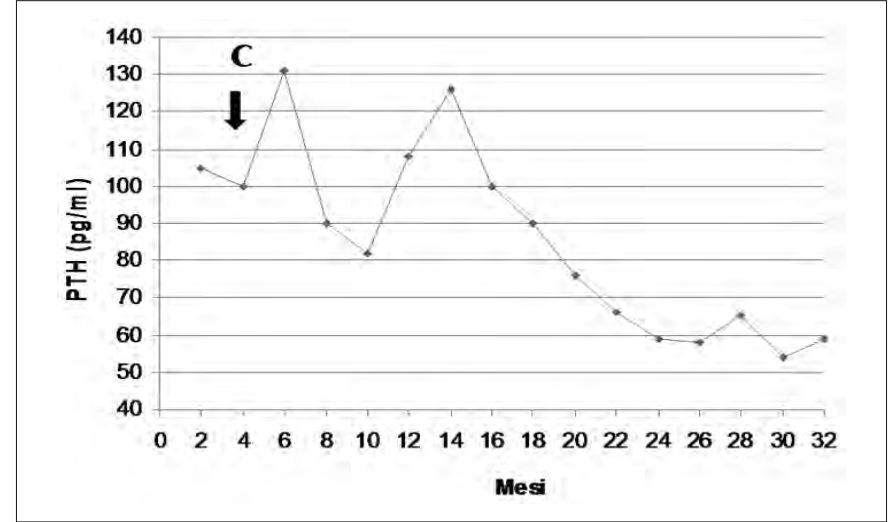

Fig. 2 - Effetti del Cinacalcet (C) sui livelli sierici di PTH durante il periodo di studio.

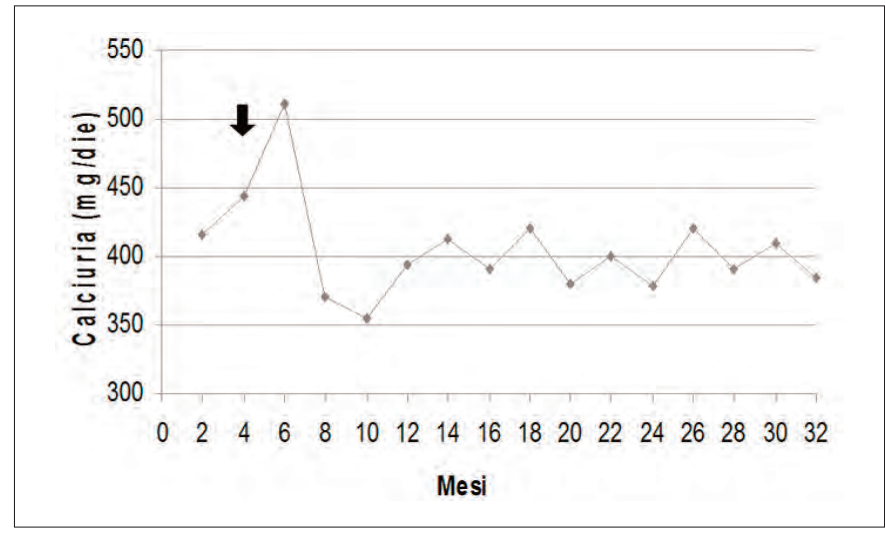

Fig. 3 - Effetti del Cinacalcet sulla escrezione urinaria di calcio durante il periodo di studio.

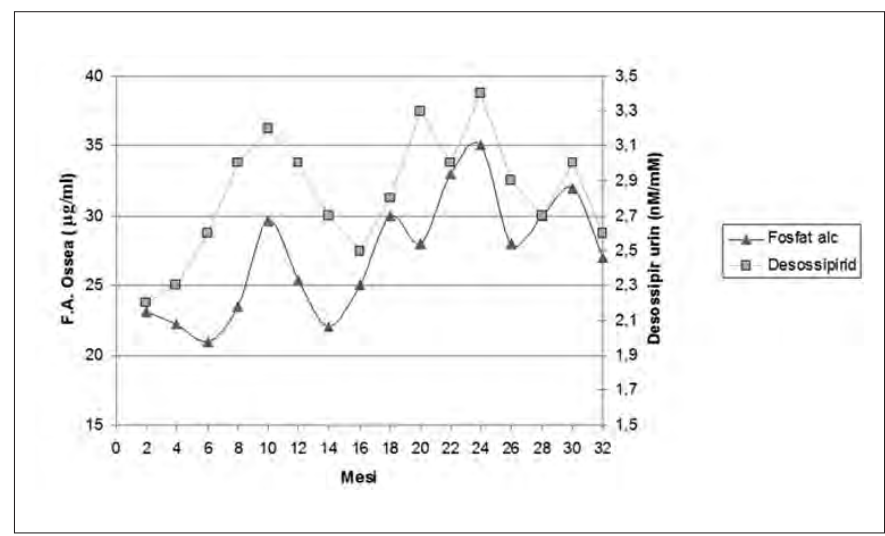

Fig. 4 - Effetti del Cinacalcet (C) sulla fosfatasi alcalina ossea e desossipiridoline urinarie durante il periodo di studio.

struttura nefrologica dove gli è stata prescritta terapia con $\mathrm{C}$ alla dose di $30 \mathrm{mg} \times 2 /$ die e gli è stata fornita campionatura gratuita per iniziare il trattamento. A tale 
data, infatti, la prescrizione del C per l'IPTP era "off label" in quanto era consentita solo per l'IPTS alla insufficienza renale cronica e per il carcinoma delle paratiroidi.

Dopo circa 4 mesi il paziente è tornato alla nostra osservazione. Gli esami da noi effettuati evidenziavano una riduzione della calcemia $(11.4 \mathrm{mg} \%)$ e del iPTH $(100 \mathrm{pg} / \mathrm{mL})$. Pertanto abbiamo consentito la prosecuzione della terapia mediante campionatura gratuita fornita dalla ditta produttrice.

L'osservazione del paziente è stata prolungata per un periodo di 32 mesi.

Il paziente non ha manifestato alcun effetto collaterale. Nella Figura 1 è riportato l'andamento dei valori di calcemia sia prima che durante la terapia con C. Nella Figura 2 sono riportate le variazioni del iPTH durante il periodo di studio. Nella Figura 3 si evidenziano le variazioni della calciuria durante il periodo di osservazione. Nella Figura 4 sono infine riportati i dati dei markers di metabolismo minerale osseo quali la fosfatasi alcalina ossea e le desossipiridoline urinarie.

\section{Caso 2}

Paziente G.A. di anni 72. La prima osservazione risale al novembre 2007. Egli perviene al nostro Centro per la Calcolosi per una storia clinica di nefrolitiasi presente da circa 30 anni. Il paziente aveva precedentemente eliminato 14 calcoli: 4 espulsi spontaneamente, 4 mediante litotomie e 6 in seguito a trattamenti di litotripsia extracorporea.

All'ecografia renale si evidenziavano 2 calcoli a $\mathrm{dx}$ di 12 e $14 \mathrm{~mm}$ e 3 calcoli a sn con un diametro tra i 5 e i 9 $\mathrm{mm}$.

È stato pertanto da noi effettuato lo studio metabolico che evidenziava la presenza di IPTP: calcemia 10.9 $\mathrm{mg} / \%$, iPTH $145 \mathrm{pg} / \mathrm{mL}$ e calciuria di $352 \mathrm{mg} /$ die. Il paziente presentava creatininemia di $1.0 \mathrm{mg} \%$ e Clcr di $65 \mathrm{~mL} / \mathrm{min}$. E stata prescritta al paziente terapia idropinica più citrati.
Nel gennaio 2008 il soggetto ha effettuato ecografia delle paratiroidi e scintigrafia paratiroidea con sestamibi, indagini che sono risultate essere nella norma. Il paziente ha poi consultato gli specialisti endocrinologi che hanno ripetuto gli esami ematochimici e strumentali da noi precedentemente effettuati. I nuovi esami di funzionalità renale hanno erroneamente evidenziato, a causa di una raccolta delle urine delle 24 ore non corretta, una insufficienza renale con $\mathrm{Clcr}<30$ $\mathrm{mL} / \mathrm{min}$. È stata così posta diagnosi di IPTS ed iniziata terapia con C $30 \mathrm{mg} /$ die.

Il paziente è tornato alla nostra osservazione dopo 6 mesi di terapia con calciomimetici. Gli esami ripetuti presso il nostro laboratorio hanno rilevato: creat. 1.1 mg\%, Clcr di $62 \mathrm{~mL} / \mathrm{min}$, calcemia 10.5 mg\%, iPTH 161 $\mathrm{pg} / \mathrm{mL}$ e calciuria $326 \mathrm{mg} / \mathrm{die}$. Ha continuato la terapia prescritta dagli endocrinologi alla dose di $30 \mathrm{mg} / \mathrm{die}$ e dopo 12 mesi di terapia gli esami laboratoristici non evidenziavano significative variazioni: calcemia 10.3 $\mathrm{mg} \%$, iPTH $150 \mathrm{pg} / \mathrm{mL}$ e calciuria $290 \mathrm{mg} / 24$ ore. Il paziente non ha manifestato alcun effetto collaterale.

\section{Discussione}

L'IPTP è abitualmente trattato mediante approccio chirurgico. La paratiroidectomia non è sempre possibile e in tali casi si deve prendere in considerazione la terapia farmacologica.

Prima dell'avvento dei calciomimetici gli unici farmaci utilizzabili nell'IPTP erano gli estrogeni e i difosfonati, pur avendo essi un effetto molto limitato sulla calcemia.

Essi agiscono principalmente inibendo il riassorbimento osseo e aiutano a limitare i danni da demineralizzazione ossea (6-8).

L'alendronato in particolare aumenta la densità ossea di circa il 3-5\% riducendo altresì i markers di turnover osseo (9).

Il C rappresenta una efficace e aggiuntiva opzione tera-

TABELLA II - EFFETTO DEL CINACALCET NELLIPTP

\begin{tabular}{|c|c|c|c|c|c|c|c|}
\hline & Dose & $\mathbf{N}^{\circ} \mathbf{p z}$ & Durata studio & Calcemia & $\mathrm{Ca}++$ & iPTH & Calciuria \\
\hline Peacock 2005 & $30-50$ & & & & & & \\
\hline Sajid-Crockett 2008 & $\begin{array}{l}\mathrm{mg} \text { bid } \\
30\end{array}$ & 78 & 13 mesi & $\downarrow 13 \%$ & $\mathrm{ND}$ & $\downarrow 7.6 \%$ & $\downarrow 13 \%$ \\
\hline Iglesias 2008 & $\begin{array}{c}\text { mg bid } \\
30\end{array}$ & 18 & 12 mesi & $\downarrow 11 \%$ & $\downarrow 13 \%$ & $\downarrow 23 \%$ & $\downarrow 13 \%$ \\
\hline
\end{tabular}


peutica inibendo la secrezione dell'ormone PTH (10). Nella Tabella II sono riportate le esperienze in letteratura sull'uso del C nell'IPTP.

La dose utilizzata è, in tutti gli studi osservati, di almeno $30 \mathrm{mg} \times 2 /$ die. A tali dosaggi i calciomimetici riducono efficacemente la calcemia, il PTH e la calciuria. Peacock et al (11) hanno valutato per 52 settimane l'efficacia del $\mathrm{C}$ nel ridurre la calcemia e il PTH studiando 78 pazienti con IPTP sottoposti a C con dose di 30-50 $\mathrm{mg} \times 2$ /die o al placebo. Il 73\% dei pazienti in terapia con $\mathrm{C}$ e il $5 \%$ dei pazienti con placebo hanno raggiunto la normocalcemia. Il PTH è diminuito del 7\% nei pazienti sotto $\mathrm{C}$ mentre è aumentato del $7 \%$ nei pazienti sotto placebo. La densità minerale ossea è risultata immodificata nei 2 gruppi. Non vi è stata differenza riguardo agli effetti collaterali. Sotto $\mathrm{C}$ si è rilevato aumento della fosforemia e del riassorbimento tubulare di fosforo. La calciuria delle 24 ore è risultata immodificata. $\grave{E}$ stato riscontrato un aumento dei markers di metabolismo minerale osseo quali la fosfatasi alcalina ossea e N-telopeptide

Sajid-Crocket et al (12) hanno studiato l'efficacia del C alla dose di $30 \mathrm{mg} \mathrm{x} 2 /$ die su 18 pazienti con IPTP. Essi hanno rilevato una normalizzazione della calcemia nel 94\% dei pazienti ma solo una modesta riduzione del PTH con una normalizzazione nel solo $25 \%$ dei casi. La calciuria non ha presentato variazioni significative. Iglesias et al (13) hanno studiato l'effetto del $\mathrm{C}$ alla dose di $30 \mathrm{mg}$ x2/die su 4 pazienti con IPTP sottoposti a intervento inefficace di paratiroidectomia. Essi hanno rilevato, dopo 1 anno di terapia, una riduzione del 10\% della calcemia, un incremento del $20 \%$ della fosforemia e una riduzione del 5.1\% del PTH. Hanno anche riscontrato una riduzione della calciuria in 3 dei 4 pazienti studiati.

Nel nostro primo paziente (Caso 1) abbiamo rilevato una riduzione della calcemia, del iPTH e della calciuria.

I dati della calciuria sotto terapia con $\mathrm{C}$ possono essere però controversi. Infatti il farmaco, riducendo la calcemia, riduce il calcio filtrato contribuendo a ridurre la calciuria. D'altro canto la caduta del PTH comporta un diminuito riassorbimento di calcio a livello del tubulo distale e quindi un'aumentata escrezione di calcio. Vi è altresì un diretto effetto del $\mathrm{C}$ sui recettori del calcio nei tubuli renali che comporta una sua aumentata escrezione (14).

Nei 32 mesi di nostra osservazione, abbiamo riscontrato un aumentato metabolismo minerale osseo, con un progressivo incremento dei markers del metabolismo osseo (Fig. 4) e concomitante diminuzione del PTH plasmatico (Fig. 2). Riscontri non dissimili sono stati rilevati da Peacock et al (11).

Una possibile spiegazione è che le fluttuazioni del PTH sierico, legate alla dose di C utilizzata, possano stimolare il turnover osseo. Non si può neanche escludere un effetto diretto del C sul turnover osseo. Sarebbero però necessari ulteriori studi al riguardo.

Il nostro secondo caso clinico (Caso 2), pur con una osservazione molto breve, sembra evidenziare l'impossibilità di qualsiasi risultato terapeutico a un dosaggio inferiore ai $30 \mathrm{mg} \mathrm{x} 2 /$ die, in accordo con i dati della letteratura (11-13). Infatti non si sono riscontrate variazioni significative dei parametri esaminati quali calcemia, PTH e calciuria nel corso dei 12 mesi di osservazione. È pur vero però che il periodo di osservazione e sicuramente minore del Caso 1 , ma comunque comparabile con gli altri della letteratura (Tab. I). I dati sul $\mathrm{C}$ non sembrano evidenziare un incremento della densità ossea (11) e non vi sono neanche dati su end point primari quali fratture, calcolosi renale o patologie cardiovascolari. Potranno essere valutati solo dopo un periodo più lungo di terapia e su casistiche più ampie. Da rilevare che non si sono manifestati effetti collaterali da $\mathrm{C}$ né nella nostra esperienza né in quelle riportate dagli altri autori.

In conclusione la terapia con C nell'IPTP rappresenta un'efficace opzione farmacologica in alternativa al trattamento chirurgico, senza significativi effetti collaterali e a un dosaggio minimo di $30 \mathrm{mg} \times 2 /$ die.

Da rilevare però l'elevato costo della terapia, con un costo mensile attuale di circa 600 Euro.

Indirizzo degli Autori:

Maurizio Terribile, MD

Unità Operativa di Nefrologia e Dialisi

Ospedale dei Pellegrini

Via Portamedina alla Pignasecca, 41

80134 Napoli

maurizio.terribile@fastwebnet.it 


\section{Bibliografia}

1. Nemeth EF, Steffey ME, Hammerland LG, et al. Calcicimetics with potent and selective activity on the parathyroid calcium receptor. Proc Natl Acad Sci USA 1998; 95: 4040-5.

2. Kawata T, Imanishi Y, Kobayashi K, et al. Direct in vitro evidence of the suppressive effect of cinacalcet HCL on parathyroid hormone secretion in human parathyroid cells withpathologically reduced calcium-sensing receptor levels. J Bone Miner Metab 2006; 24: 300-06.

3. Block GA, Martin KJ, de Francisco AL, Turner SA, Avram MM, Suranyi MG, et al. Cinacalcet for secondary hyperparathyroidism in patients receiving hemodialysis. N Engl J Med 2004; 350(15): 1516-25.

4. Lindberg JS, Culleton B, Wong G, Borah MF, Clark RV, Shapiro $\mathrm{WB}$, et al. Cinacalcet $\mathrm{HCl}$, an oral calcimimetic agent for the treatment of secondary hyperparathyroidism in hemodialysis and peritoneal dialysis: a randomized, double-blind, multicenter study. J Am Soc Nephrol 2005; 16(3): 800-7.

5. Bilezikian JP, Potts JT, Fuleihan G, et al. Summary statement from a workshop on asymptomatic primary hyperparathyroidism: a perspective for the 21 st century. J Clin Endocrinol Metab 2002; 87: 5353-61.

6. Selby PL, Peacock M. Ethinyl estradiol and norethindrone in the treatment of primary hyperparathyroidism in postmenopausal womem. N Engl J Med 1996; 314: 1481-85.

7. Chow CC, Chan WB, Li JK, et al. Oral alendronate increases bone mineral density in postmenopausal women with primary hyperparathyroidism. J Clin Endocrinol Metab 2003; 88: 581-87.

8. Orr-Walker BJ, Evans MC, Clearwater JM, Horne A, Grey AB, Reid IR. Effects of hormone replacement therapy on bone mineral density in postmenopausal women with primary hyperparathyroidism: four-year follow-up and comparison with healthy postmenopausal women. Arch Intern Med 2000; 160(14): 2161-6.

9. Hassani S, Braunstein GD, Sebel MJ, et al. Alendronate therapy of primary hyperparathyroidism. Endocrinologist 2001; 11: 459-64.

10. Vestergaard P. Current pharmacological option for the management of primary hyperparathyroidism. Drugs 2006: 66: 2189-211.

11. Peacock M, Bilezikian JP, Klassen PS, Guo MD, Turner SA, Shoback D. Cinacalcet hydrochloride maintains long-term normocalcemia in patients with primary hyperparathyroidism. J Clin Endocrinol Metab 2005; 90(1): 135-41.

12. Sajid-Crockett S, Singer FR, Hershman JM. Cinacalcet for the treatment of primary hyperparathyroidism. Metabolism 2008; 57 (4): 517-21.

13. Iglesias P, Ais G, González A, Tajada P, Garc'a Arévalo C, Fernández Pardo E, D'ez JJ. Acute and one-year effects of cinacalcet in patients with persistent primary hyperparathyroidism after unsuccessful parathyroidectomy. Am J Med Sci 2008; 335(2): 111-4

14. Riccardi D, Hall AE, Chattopadhyay N, et al. Localization of the extracellular CA2+/polyvalent cation-sensing protein in rat kidney. Am J Physiol 1998; 274: F611-22. 